\title{
Editorial
}

\section{Ecosystemic Evolution Fed by Smart Systems}

\section{Dino Giuli}

Department of Information Engineering (DINFO), University of Florence, Via Santa Marta, 3, Florence 50139, Italy; dino.giuli@unifi.it

Received: 5 March 2018; Accepted: 6 March 2018; Published: 10 March 2018

Information Society is advancing along a route of ecosystemic evolution. ICT and Internet advancements, together with the progression of the systemic approach for enhancement and application of Smart Systems, are grounding such an evolution. The needed approach is therefore expected to evolve by increasingly fitting into the basic requirements of a significant general enhancement of human and social well-being, within all spheres of life (public, private, professional). This implies enhancing and exploiting the net-living virtual space, to make it a virtuous beneficial integration of the real-life space. Meanwhile, contextual evolution of smart cities is aiming at strongly empowering that ecosystemic approach by enhancing and diffusing net-living benefits over our own lived territory, while also incisively targeting a new stable socio-economic local development, according to social, ecological, and economic sustainability requirements. This territorial focus matches with a new glocal vision, which enables a more effective diffusion of benefits in terms of well-being, thus moderating the current global vision primarily fed by a global-scale market development view.

Basic technological advancements have thus to be pursued at the system-level. They include system architecting for virtualization of functions, data integration and sharing, flexible basic service composition, and end-service personalization viability, for the operation and interoperation of smart systems, supporting effective net-living advancements in all application fields. Increasing and basically mandatory importance must also be increasingly reserved for human-technical and social-technical factors, as well as to the associated need of empowering the cross-disciplinary approach for related research and innovation. The prospected eco-systemic impact also implies a social pro-active participation, as well as coping with possible negative effects of net-living in terms of social exclusion and isolation, which require incisive actions for a conformal socio-cultural development. In this concern, speed, continuity, and expected long-term duration of innovation processes, pushed by basic technological advancements, make ecosystemic requirements stricter. This evolution requires also a new approach, targeting development of the needed basic and vocational education for net-living, which is to be considered as an engine for the development of the related 'new living know-how', as well as of the conformal 'new making know-how'.

The papers of the special issue are significant contributions samples within the general ecosystemic view above highlighted. The first group of papers ([1-5]) pertain the focus on human and social factors through interdisciplinary and transdisciplinary approaches, which look for enhancing quality of life supported by the smart system environment.

The second group of papers ([6-9]) pertains to some relevant technological infrastructure enhancements, based on exploitation of the Internet of Things the 5G network paradigms.

Several contributed papers widely point out also the relevance of smart city context for grounding and developing the ecosystemic approach, through advancement of both intangible and tangible infrastructures. Contributions made by each paper is below outlined.

The first paper-by Martelli [1] — exposes and grounds an initiative point of view about new education needed for smart citizenship. Education has to cope indeed with citizen awareness lack. The paper's primary concern is about privacy and freedom requirements connected with the knowledge bases generated by the IT services. For such a purpose, an original education methodology 
is prospected in the paper, while also reporting and discussing related experiences made through some specifics use cases.

The second paper—by Angelini, Carrino, Khaled, Riva-Mossman, and Mugellini [2]—describes and discusses a new transdisciplinary research platform jointly developed by four universities to support co-creation of ICT based innovative products, services, and practices, to fit into user requirements of older adults. Such a platform is devised as the Senior Living Lab (SLL), by exploiting cooperative transdisciplinary support of designers economists, engineers, and healthcare professionals, as well as pro-active participation of end users (older adults). The paper describes the approach thus adopted for the lab's operation, as well reporting objectives and operational outcomes of some current projects concerned with healthy nutrition to cope with frailty, improved autonomous mobility, and social communication to prevent isolation.

The third paper-by Marti, Megens, and Hummels [3] — exploits an ecosystemic view which focuses on the needed advancements of "user-centered design" for social innovation. Paper focus is needed on advanced methodologies for transforming data-driven design towards data-enabled participatory and co-creative design. The new methodology purposely devised to design smart systems, called experimental design landscapes (EDL), is presented in this paper, together with two samples projects. Such projects are re4specively concerned with the following topics: human behavior change mediated by sensing technologies; social platform to sustain new processes of deliberative democracy.

The fourth paper-by Lettieri [4] — is a significant contribution on current advancements and perspectives of computational social sciences (CSS) for evolution of policy design and rule making within smart societies. While providing the needed review, the paper highlights and discusses promising scientific advancements, which can be prospected for CSS valuing. The primary focus is on the new mechanism needed for regulating both formulation and evolution towards a smart society.

The fifth paper-by Guidi, Miniati, Mazzolla, and Iadanza [5] - pertains to smart health systems application. This contribution is concerned with the use of a cloud platform to provide analytics-as-a-service (AaaS) tools, for heart disease continuous monitoring and fast detection. Application and experimentations are described in the paper, which have been developed through personalization of basic IBM Watson Analytics tools, while exploiting only electrocardiographic signal and heart rate variability, for monitoring and heart failure disease detection. Based on experimental outcomes, advantages and drawbacks of the cloud approach with respect to the usual static approach are discussed in the paper.

The sixth paper-by Carrino, Mugellini Khaled, Ouerhani, and Ehrensberger [6]—is a significant contribution concerned with advancements of Internet of Things for Urban Innovation (iNUIT), as a key support for smart city development. The paper's contributions are framed within a specific multi-year research program started in Switzerland. Reported research activities and results refers to two started projects which are included in such a program. The first project (smart crowd) is concerned with monitoring the crowd's movement to detect possible dangerous scenarios. Purposeful, real-time tracking is afforded through sensors which are available within smart-phones. The second project $(\mathrm{OpEc})$ is concerned with exploitation of an Internet of Things approach, to implement dynamic street light management and control, aiming at street light energy saving. Shown experimental results of both projects point out efficacy of the adopted solutions.

The seventh paper—by Paradiso, Paganelli, and Giuli [7]—is contributing to the enhancements of "Internet of Things" approach aimed at energy consumption cost saving in residential places. The paper is specifically proposing and evaluating a new non-intrusive load monitoring (NILM) instrumental asset, keeping the needed capabilities for monitoring and control of energy consumption while reducing instrumental complexity and cost of the current load monitoring solutions. The proposed NILM solution is based on: (i) disaggregation of whole-house consumption into the single portions associate to each consuming device; (ii) exploiting information of users presence and hourly use of appliances; (iii) enabling a constructive behavior of final user for energy saving; (iv) addressing monitoring for total active power measurements, which can be sampled at a much lower frequency 
thus reducing their data set size. As shown in the paper, positive results have been obtained through simulations fed through and experimental open dataset.

The eighth paper-by Martini and Paganelli [8]—is concerned with emerging network technologies based on the paradigms of software-defined networks (SDN) and network function virtualization (NFV), which are aimed at enhancement of network operation flexibility and cost reduction. Such an evolution is particularly relevant for the current roadmap of the 5G network and for its impact in smart city contexts. In such a context, criticalities and limitations currently emerge for cross-virtualization and dynamic integration of network services among different network operators. The paper contribution is concerned with such a problem. A new approach is proposed and discussed, which is based joint exploitation of the service-oriented architecture (SOA) paradigm, to cope with the multi-operator virtual network integration, thus complementing the current NFV and SDN approaches. A new network architecture is thus worked out and discussed. Preliminary results of prototype implementation and testing activities are also presented, which highlights also benefits for network service providers.

The ninth paper-by Fantacci and Marabissi [9]—is concerned with advancements of telecommunications technologies for wireless communications. The topics faced pertain to the current roadmap of the $5 \mathrm{G}$ network, which are particularly important for related critical requirements arising within the smart city context. A specific contribution is indeed made about a new methodology to be adopted to improve usage of all potential spectrum resources. Resorting to cognitive radio technology is prospected and discussed, in order to support context-aware dynamic optimization of the spectrum usage and sharing. A review is made on such a subject, by exploiting two relevant new network paradigms: heterogeneous networks and Machine-to-Machine communications.

Acknowledgments: The guest editors wish to thank all the contributing authors, the professional reviewers for their precious help with the review assignments, and the excellent editorial support from the Future Internet journal at every stage of the publication process of this special issue.

Conflicts of Interest: The authors declare no conflict of interest.

\section{References}

1. Martelli, C. A point of view on New Education for Smart Citizenship. Future Internet 2017, 9, 4. [CrossRef]

2. Angelini, L.; Carrino, S.; Khaled, O.A.; Riva-Mossman, S.; Mugellini, E. Senior Living Lab: An Ecological Approach to Foster Social Innovation in Ageing Innovation. Future Internet 2016, 8, 50. [CrossRef]

3. Marti, P.; Megens, C.; Hummels, C. Data Enabled Design for Social Change: Two Case Studies. Future Internet 2016, 8, 46. [CrossRef]

4. Lettieri, N. Computational Social Science, the Evolution of Policy Design and Rule Making in Smart Societies. Future Internet 2016, 8, 19. [CrossRef]

5. Guidi, G.; Miniati, R.; Mazzola, M.; Iadanza, E. Case Study: IBM Watson Analytics Cloud Platform as Analytic-as-aServbice System for Hearth Failure Early Detection. Future Internet 2016, 8, 32. [CrossRef]

6. Carrino, F.; Mugellini, E.; Abou Khaled, O.; Ouerhani, N.; Ehrensberger, J. iNuit: Internet of Things for Urban Innovation. Future Internet 2016, 8, 18. [CrossRef]

7. Paradiso, F.; Paganelli, F.; Giuli, D.; Capobianco, S. Context-Base Enertgy Disaggregation in Smart Homes. Future Internet 2016, 8, 4. [CrossRef]

8. Martini, B.; Paganelli, F. A Service-Oriented Approach for Dynamic Chaining of Virtual Network Functions over Multi-Provider Software-Defined Networks. Future Internet 2016, 8, 24. [CrossRef]

9. Fantacci, R.; Marabissi, D. Cognitive Spectrum Sharing: An Enabling Wireless Communication Technology for a Wide Use of Smart Systems. Future Internet 2016, 8, 23. [CrossRef] 